\title{
STABILITÉ TEMPORELLE DES STRUCTURES SPATIALES DES PEUPLEMENTS PISCICOLES DES RÉGIONS LANGUEDOC-ROUSSILLON ET PROVENCE-ALPES-CÔTE D'AZUR.
}

\author{
L. BLANC (1) et D. BEAUDOU (2)
}

(1) UMR - CNRS 5556, Laboratoire d'Hydrobiologie Marine et Continentale, case courrier 093, Université Montpellier II, place Eugène Bataillon, 34095 Montpellier Cedex 5, France.

(2) Délégation Régionale du CSP $n^{\circ} 8,55$ chemin du Mas de Matour, 34790 Grabels, France.

\section{RÉSUMÉ}

Les peuplements piscicoles de 35 stations des régions Languedoc-Roussillon et Provence-Alpes-Côte d'Azur ont été échantillonnés sur quatre années entre 1993 et 1996, dans le cadre du Réseau Hydrobiologique et Piscicole mis en place par le Conseil Supérieur de la Pêche. Pour analyser ces données de type espèces-stations-dates, nous avons utilisé deux méthodes d'analyse multitableaux : l'analyse triadique partielle (THIOULOUSE et CHESSEL, 1987 ; BLANC et al., 1998) et l'Analyse Factorielle des Correspondances de FOUCART $(1978,1983)$. Quelle que soit la méthode utilisée, une structure spatiale espèces-stations commune aux différentes dates a été mise en évidence. La première oppose principalement des stations à truite aux stations à cyprinidés et révèle un double effet de régionalisation. La deuxième a mis en évidence une structure amont-aval proche de celles des schémas ichtyologiques de référence (HUET, 1954 ; VERNEAUX, 1973, 1981). L'étude temporelle des structures autour de la structure moyenne a montré une forte stabilité durant la période étudiée.

Mots-clés : poissons, analyse triadique partielle, AFC de FOUCART, évolution temporelle, région méditerranéenne.

\section{TEMPORAL STABILITY OF SPATIAL STRUCTURES OF FISH COMMUNITY IN LANGUEDOC-ROUSSILLON AND PROVENCE-ALPES-CÔTE D'AZUR REGIONS.}

\section{ABSTRACT}

Fish community of 35 stations of both Languedoc-Roussillon and Provence-AlpesCôte d'Azur regions (France) were sampled from 1993 to 1996 during the survey program "Réseau Hydrobiologique et Piscicole " conducted by the Conseil Supérieur de la Pêche. The species-station-date data set was analysed using two K-tables (4 dates) statistical methods: Partial Triadic Analysis (THIOULOUSE and CHESSEL, 1987 ; BLANC et al., 1998) and series of contingency tables (FOUCART, 1978, 1983). With both methods, results suggested that there was a common spatial structure for all dates of sampling. The first method revealed an opposition between stations characterized by trout and stations 
where Cyprinidae species were dominant. The second method highlighted an upstreamdownstream structure of fish community similar to those considered as references (HUET, 1954 ; VERNEAUX, 1973, 1981). The analysis of temporal variation around the compromise structure showed that fish assemblages were stable over the studied period.

Key-words : fish, partial triadic analysis, multiple series of contingency tables, temporal evolution, Mediterranean region.

\section{INTRODUCTION}

L'étude des peuplements piscicoles est depuis longtemps au coeur des préoccupations des scientifiques. Déjà HUET en 1954 puis VERNEAUX en 1973 et plus récemment HUGUENY (1990), BOËT et al. (1991), OBERDOFF et al. (1993) ou CHANGEUX (1995) se sont intéressés à la structure et à la répartition des espèces. VERNEAUX (1981) et GRANDMOTTET (1983) ont ainsi montré que les poissons pouvaient servir d'indicateur pour apprécier l'état général des cours d'eau. Suite à cette constatation, les gestionnaires ont donc de plus en plus besoin d'accroître leurs connaissances sur les peuplements ichtyologiques de l'ensemble du réseau hydrographique français.

Dans le cadre du Réseau Hydrobiologique et Piscicole (RHP), le Conseil Supérieur de la Pêche (CSP) a mis en place, à l'échelle nationale, un réseau de suivi piscicole annuel sur un nombre important de stations. Un des objectifs principaux de ce réseau est de disposer d'un bilan annuel de l'état des peuplements piscicoles et d'en suivre l'évolution. Sur une station donnée, l'état d'un peuplement est apprécié en analysant la composition spécifique du peuplement observé par rapport au peuplement attendu en fonction du milieu, aux exigences des espèces présentes et en étudiant la structure des différentes populations. A la fois dans l'analyse stationnelle et dans l'analyse globale, deux questions se dégagent : peut-on mettre en évidence une structure spatiale ? Quelle est la stabilité temporelle de cette structure ? Ces questions se posent de façon encore plus aiguë pour les peuplements de poissons du pourtour du bassin méditerranéen dont le caractère distinctif est reconnu depuis longtemps (CHANGEUX et PONT, 1995). Cependant, comme le soulignent ces mêmes auteurs, ni les classifications établies par ILLIES et BOTOSANEANU (1963), ni la méthode développée par VERNEAUX (1973) ne peuvent être directement utilisables pour les cours d'eau méridionaux puisqu'elles ne prennent pas en compte les particularités de cette région (températures estivales plus élevées, irrégularité des débits...). Pour répondre aux questions posées, il est donc nécessaire de disposer d'outils d'analyse adaptés. Les méthodes d'analyse multitableaux comme l'analyse triadique partielle (THIOULOUSE et CHESSEL, 1987 ; BLANC et al., 1998) ou l'Analyse Factorielle des Correspondances de FOUCART (1978, 1983), permettent d'aborder de telles données tri-dimensionnelles de type espèces-stations-dates.

En appliquant ces méthodes d'analyse aux données issues du RHP concernant les régions Languedoc-Roussillon et Provence-Alpes-Côte d'Azur, l'objectif de cet article est de chercher une typologie spatiale espèces-stations commune aux différentes dates de suivi et d'en étudier la stabilité temporelle.

\section{MATÉRIEL ET MÉTHODES}

\section{Données utilisées}

II s'agit de données décrivant le peuplement piscicole de stations réparties sur 11 départements des régions Languedoc-Roussillon (LR) et Provence-Alpes-Côte d'Azur (PACA). Ces stations ont été échantillonnées une fois par an, à la même saison (été), 
entre 1993 et 1996 dans le cadre du RHP. Dans un premier temps, nous avons constitué un ensemble de données homogènes en ne conservant que les stations prospectées par pêche électrique à pied sur toute la largeur avec deux passages successifs. Cette contrainte d'homogénéité des données, afin de pouvoir adopter une stratégie d'ACP, nous a conduit à écarter les zones où une prospection complète est impossible (parties médianes et aval des grands cours d'eau). Par ailleurs, l'analyse triadique partielle impose d'utiliser les mêmes variables mesurées sur les mêmes stations à plusieurs reprises. Nous avons donc également écarté les stations pour lesquelles une des prospections annuelles sur les 4 années n'a pu être réalisée en raison de mauvaises conditions hydrologiques. Ces deux contraintes aboutissent à la sélection de 35 stations (Tableau I). Ces stations sont réparties de façon relativement homogène sur l'ensemble des 2 régions, à l'exception des parties basses des grands cours d'eau (Figure 1).

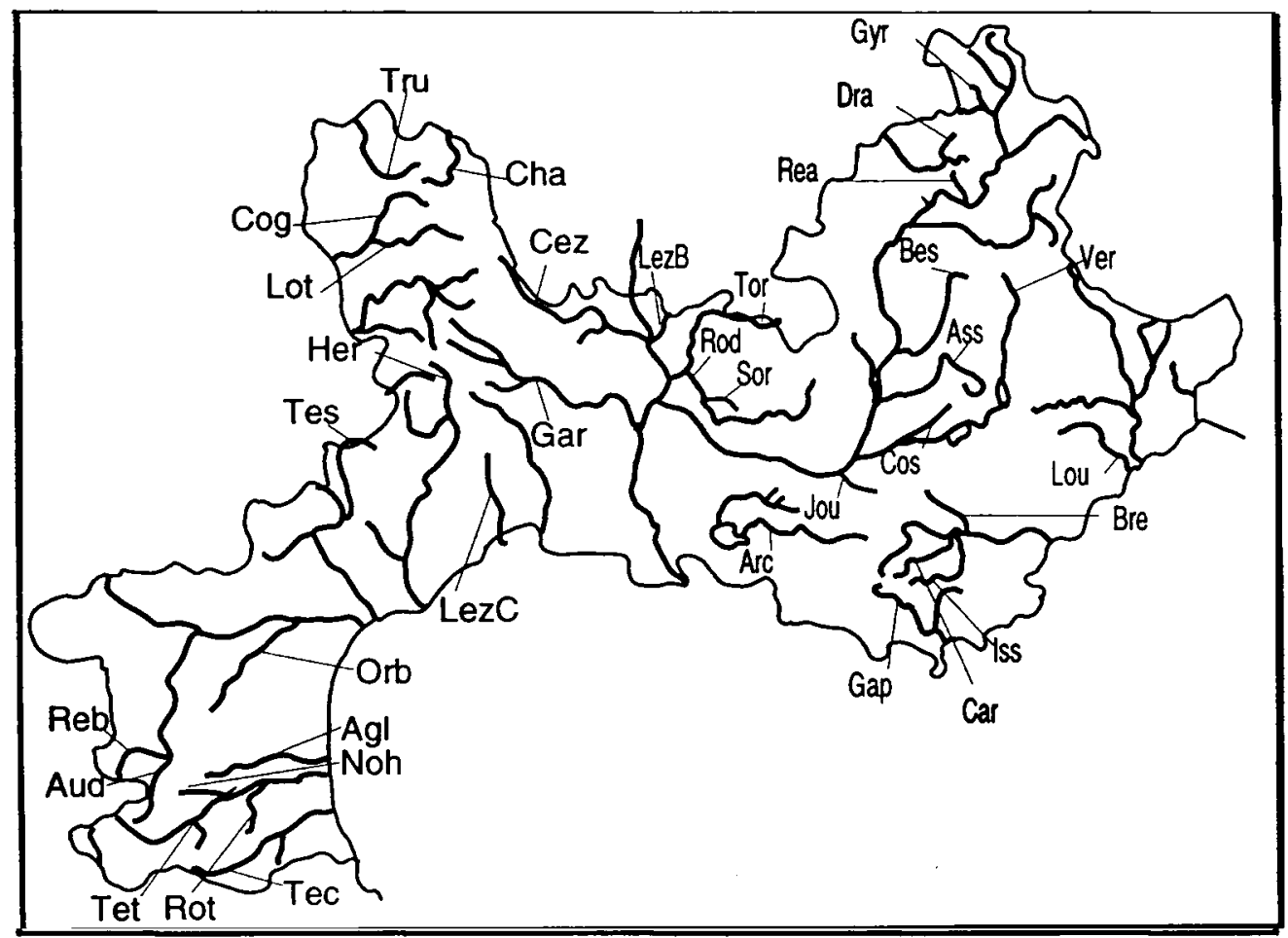

Figure 1

Stations d'échantillonnage des régions Languedoc-Roussillon (LR) et ProvenceAlpes-Côte d'Azur (PACA) (les codes des stations sont dans le Tableau I).

\section{Figure 1}

Sampling stations of the Languedoc-Roussillion and the Provence-Alpes-Côte d'Azur regions (see Table I for stations codes).

Sur les 35 stations retenues, 28 espèces ont été recensées. Seulement 21 ont été retenues pour l'analyse. En effet, les espèces de très faible occurrence, c'est-à-dire présentes sur moins de $3 \%$ de la totalité des inventaires (soit une ou deux années sur une ou deux stations), ont été supprimées. Il s'agit du brochet, de la lamproie de Planer, du poisson-chat, du Pseudorasbora parva, du saumon de fontaine, de la truite arc-en-ciel et de la tanche. La présence de certaines de ces espèces est liée à des déversements destinés à la gestion halieutique (truite arc-en-ciel) ou qui peuvent être accidentels (Pseudorasbora parva). Certaines espèces déversées (brochet, tanche) peuvent se maintenir par la suite en faibles effectifs dans des stations dont les caractéristiques mésologiques, et notamment les habitats, ne permettent cependant pas leur pérennisation. 


\section{Tableau I}

Codes des espèces et des stations d'échantillonnage retenues pour l'analyse.

\section{Table I}

Species and sampling stations codes for the analysis.

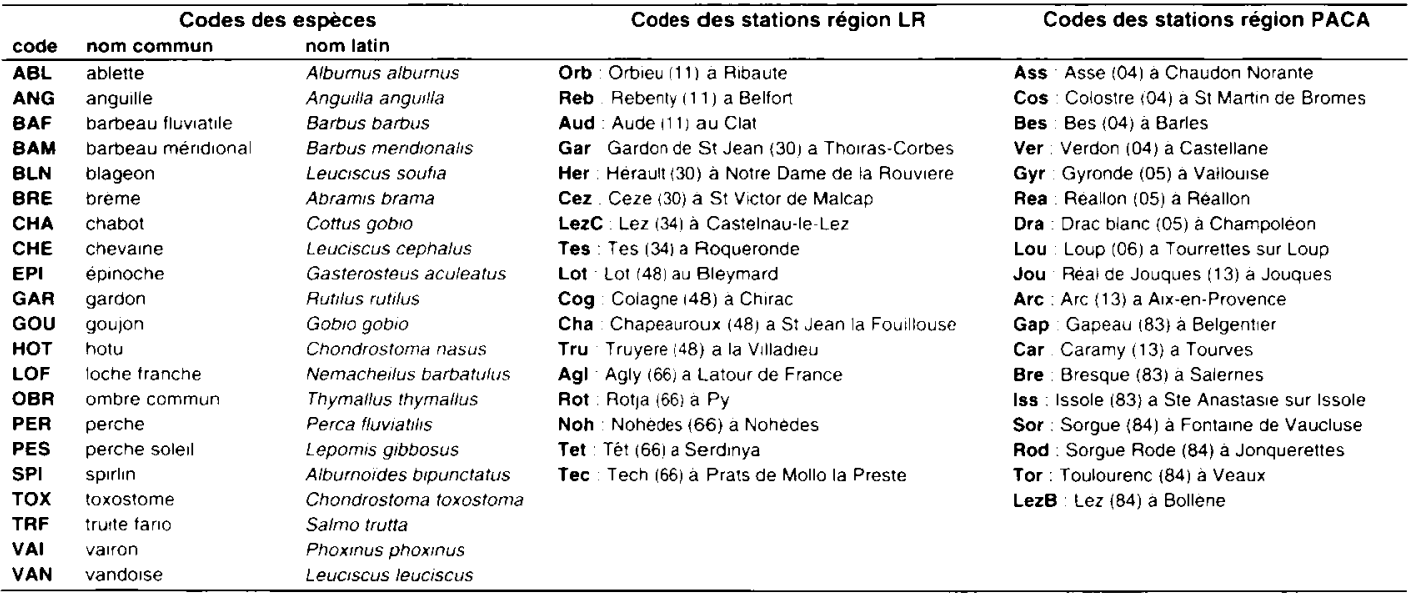

Biomasses et densités sont deux variables utilisées conjointement (FISCHER et ECKMANN, 1997) ou alternativement (LOUIS et al. (1995), LAROCHE et al. (1997) pour la biomasse, CARREL et al. (1995) pour la densité). Les données traitées ici sont des biomasses estimées pour chaque espèce par la méthode de DE LURY (1947), exprimées en $\mathrm{g}$ pour $100 \mathrm{~m}^{2}$. Nous avons retenu la biomasse par unité de surface plutôt que la densité car elle exprime mieux la productivité du milieu et donne une meilleure idée de l'importance relative de chaque espèce. De plus, elle permet de s'affranchir des fluctuations de l'efficacité de pêche sur les juvéniles de cyprinidés de très petites tailles.

On obtient ainsi pour chaque année: 1993, 1994, 1995 et 1996, un tableau à 35 lignes-stations et 21 colonnes-espèces.

\section{Analyse de données}

Au préalable, les données subissent une transformation log : $y=\log (x+1)$ afin d'homogénéiser les variances et de minimiser les effets de non normalité.

Face à un tableau de données faunistiques, on a principalement le choix entre deux méthodes d'analyses multivariées classiques : l'Analyse Factorielle des Correspondances (AFC) où la transformation effectuée correspond à un double centrage (NOY-MEIR, 1973) et l'Analyse en Composantes Principales (ACP) centrée par espèce (chaque espèce a une moyenne nulle). Cette question est discutée par DOLÉDEC et CHESSEL (1991). Nous justifions dans un premier temps le choix de l'ACP plutôt que de l'AFC pour deux raisons : d'une part, cette méthode s'avère plus adaptée aux données quantitatives dont nous disposons (biomasses) et d'autre part, dans la mesure où nous avons sélectionné uniquement les pêches électriques à pied avec deux passages successifs, nous pouvons considérer l'échantillonnage comme standardisé, ce qui nous autorise à utiliser l'ACP. L'analyse triadique partielle (THIOULOUSE et CHESSEL, 1987 ; BLANC et al., 1998) va nous permettre de faire des ACP simultanées et coordonnées des quatre tableaux faunistiques. L'objectif de cette méthode est de trouver une structure faunistique spatiale commune à toutes les années et d'étudier la stabilité temporelle autour de cette structure moyenne. La méthode s'appuie d'abord sur la recherche d'une moyenne de tableaux appelée " compromis " que l'on analyse, puis sur la reproductibilité du compromis par chacun des tableaux initiaux. 
Cependant, il est également intéressant d'aborder une stratégie d'AFC qui est la méthode la plus fréquemment employée pour traiter un tableau de données d'abondance de poissons (VERNEAUX, 1973, 1981 ; BOËT et al., 1991 ; OBERDOFF et al., 1993 ; CHANGEUX, 1995). En effet, l'AFC ordonne les taxons et les stations en fonction de leur profil, elle met en évidence des associations d'espèces indépendamment de leur abondance. Ainsi, alors que l'ACP prend en compte les variations d'abondance d'espèces, le double centrage de l'AFC les élimine. Cette méthode revalorise le rôle des espèces rares, qui peuvent avoir un intérêt d'un point de vue écologique, dans la constitution des axes factoriels. L'AFC proposée par FOUCART (FOUCART, 1978, 1983) permet de traiter simultanément les quatre tableaux faunistiques dont nous disposons. Sa mise en oeuvre se fait de façon analogue à l'analyse triadique partielle : recherche d'un compromis (d'une moyenne), analyse du compromis et analyse de chacun des tableaux autour de la structure moyenne. Le principe général de cette méthode a été décrit par BLANC et al. (1998). Elle consiste à construire un compromis en prenant une moyenne uniformément pondérée des tableaux et à faire l'AFC de ce compromis qu'on interprète comme toute analyse des correspondances classique. Pour étudier l'écart autour de la structure du compromis, les axes, les lignes et les colonnes des $K$ tableaux initiaux sont projetés en individus supplémentaires.

Ces deux analyses présentent donc une forte complémentarité, aussi nous les avons appliquées toutes les deux aux données piscicoles du RHP. Les analyses ainsi que les représentations graphiques ont été effectuées avec le logiciel ADE-4 (THIOULOUSE et al., 1997) librement diffusé sur Internet (http://pbil.univ-lyon1.fr/ADE-4/ADE-4F.html).

\section{RÉSULTATS ET DISCUSSION}

\section{ACP séparées des 4 tableaux}

Dans un premier temps, une ACP centrée par colonne est effectuée sur chacun des tableaux afin d'avoir un aperçu des résultats correspondant aux différentes années. Les histogrammes des valeurs propres (Figure $2 \mathrm{~A}$ ) montrent que le premier axe est le plus informatif puisqu'il représente de 39,9 à $43,7 \%$ de l'inertie totale du tableau suivant les années. Bien que les histogrammes suggèrent d'étudier au moins quatre axes pour obtenir une large information, nous limiterons ici l'analyse à deux axes, l'idée étant plus d'avoir un aperçu des analyses séparées que de détailler chacune d'entre elles. Les Figures $2 \mathrm{~B}$ et $2 \mathrm{C}$ représentent respectivement la position des espèces et des stations dans le plan 1-2 des différentes analyses. L'axe 1 oppose la truite fario et le chevaine. Ces deux espèces ont les occurrences les plus importantes dans les tableaux de données (cf. Annexe). En effet, sur les 35 stations retenues et au moins trois années sur quatre, la truite est présente dans trente stations et le chevaine dans dix-sept. L'axe 2 ordonne les différentes espèces entre un pôle constitué par le barbeau méridional, le blageon, le goujon et un pôle représenté par le barbeau fluviatile en 1993 et 1995 ou l'anguille en 1994 et 1996.

D'une année à l'autre, cette organisation générale se retrouve plus ou moins, d'où l'intérêt de coordonner ces quatre analyses par l'analyse triadique partielle afin d'en obtenir une représentation commune.

\section{Analyse triadique partielle des 4 tableaux faunistiques}

\section{Recherche et analyse du compromis}

La matrice des corrélations vectorielles RV (Tableau II) entre les tableaux-dates montre l'existence de corrélations très importantes entre les tableaux. La corrélation la plus 

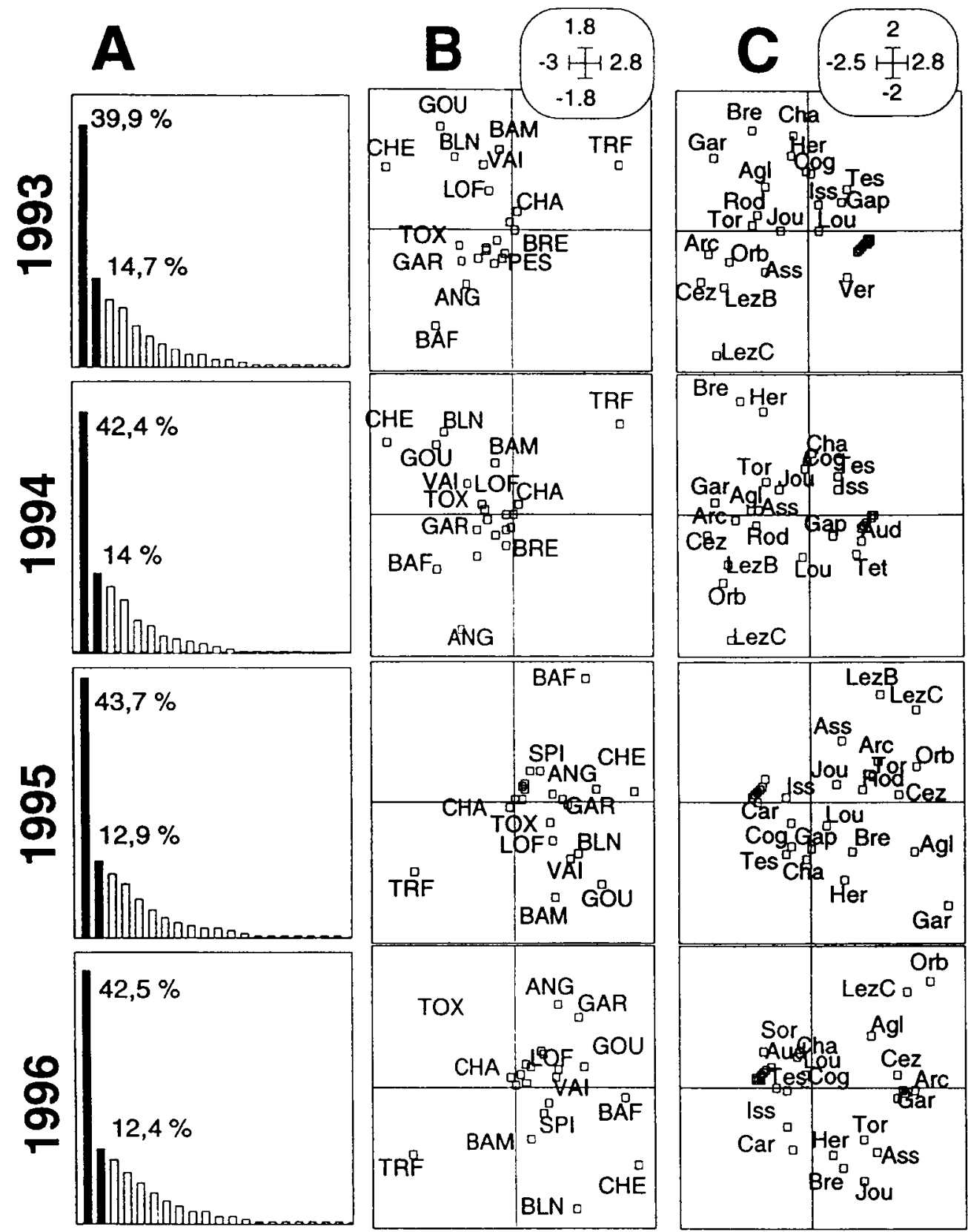

\section{Figure 2}

ACP centrées de chacun des tableaux (années). (A) Histogramme des valeurs propres. (B) Projection des espèces de poissons sur les axes factoriels 1 et 2 des analyses. (C) Projection des stations sur les axes factoriels 1 et 2 des analyses. Les codes des espèces et des stations sont dans le Tableau I.

\section{Figure 2}

Centered PCAs of each table (years). (A) Histogram of eigenvalues. (B) Projection of fish taxa on the principal axes 1 and 2. (C) Projection of stations on the principal axes 1 and 2 . See Table $I$ for stations and species codes. 
forte est observée entre 1995 et $1996(0,903)$ et la plus faible entre 1993 et $1996(0,797)$. La diagonalisation de la matrice de covariances vectorielles (Covv) fournit les axes principaux (Figure 3A), dont le premier représente à lui seul $89,9 \%$ de l'inertie totale. On retient essentiellement ce premier axe qui a une fonction de définition d'une moyenne alors que les suivants caractérisent l'écart au modèle moyen. La projection de chaque tableau sur l'axe 1 donne un aperçu de la ressemblance entre les tableaux (Figure 3B). Les 4 tableaux ont des coordonnées quasiment identiques sur cet axe, ce qui suggère qu'ils ont donc des structures très semblables.

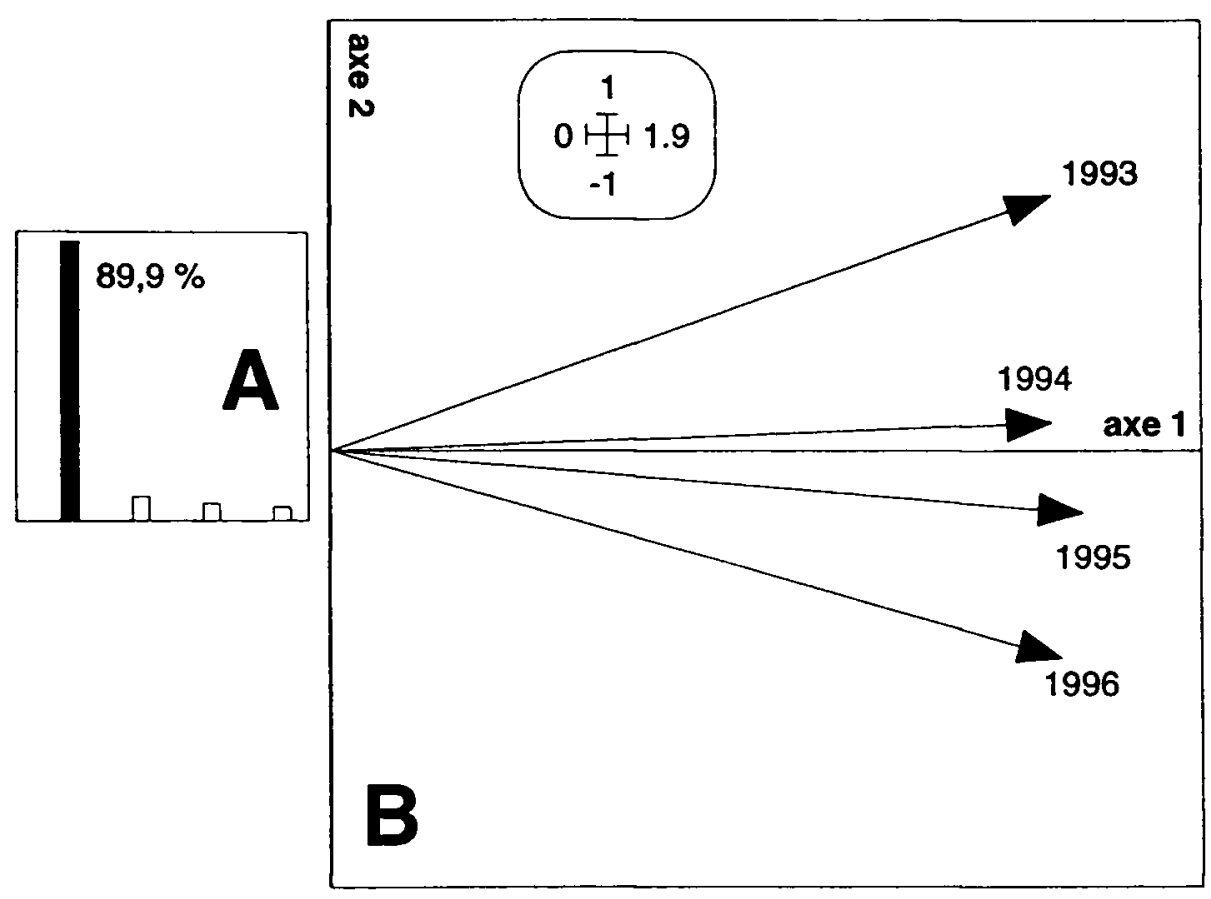

Figure 3

Analyse triadique partielle : interstructure. (A) Histogramme des valeurs propres issues de la diagonalisation de la matrice des covariances vectorielles. (B) Projections des quatre tableaux (années) sur les axes 1 et 2 de l'interstructure.

\section{Figure 3}

Partial triadic analysis : interstructure. (A) Histogram of eigenvalues generated by the diagonalization of the matrix that contains covariances of the data sets. (B) Projections of the four data sets (years) on the interstructure axes 1 and 2.

Ce sont les composantes du premier vecteur propre normé $(89,9 \%$ de l'inertie) qui fournissent les coefficients de pondération $\alpha_{k}$ (Tableau II) affectés à chacun des tableaux. Ces coefficients représentent le poids du tableau $k$ dans la définition du compromis. Leurs valeurs très semblables $(0,493 ; 0,494 ; 0,514 ; 0,499)$ montrent que les 4 tableaux participent de façon égale à la construction du compromis. La somme des 4 tableaux $\left(1993,1994,1995\right.$ et 1996) pondérée par les poids $\alpha_{k}$ constitue le tableau compromis. On analyse ce compromis comme une analyse d'inertie standard. L'histogramme des valeurs propres issues de cette analyse (Figure 4A) montre que l'axe 1 est prédominant et explique $45,2 \%$ de l'inertie du tableau. Les trois axes suivants représentent une part plus faible de l'inertie : de 13,4 à 9,6\%. Nous prendrons en compte par la suite ces 4 axes afin de disposer d'une quantité d'informations jugée suffisante $(78,7 \%$ de l'information initiale). 


\section{Tableau II}

Paramètres numériques (indices typologiques) associés à l'analyse triadique partielle des données du RHP. RV = matrice des coefficients de corrélations vectorielles entre tableaux; $\alpha_{k}=$ pondération indiquant la participation du tableau $\boldsymbol{k}$ à la définition du compromis ; Var. vect. = variance vectorielle mesurant l'inertie interne du tableau $k$; $\operatorname{Cos}^{2}$ : cosinus carré entre le tableau $k$ et le compromis réduit à sa partie interprétée, ici suivant quatre axes.

\section{Table II}

Numerical parameters (typology indexes) associated with partial triadic analysis processed on the RHP data. RV = correlation matrix between tables ; $\alpha_{k}=$ weights of tables in the definition of the compromise ; Var. vect. = square norm of data set measuring the internal inertia of the $k$-th table; $\operatorname{Cos}^{2}:$ square cosine between a data set and the approximated compromise using four axes.

\begin{tabular}{clllcccc}
\hline Tableau & \multicolumn{3}{c}{ RV } & Poids $\left(\alpha_{k}\right)$ & Var. vect. & Cos $^{2}$ \\
\hline 1993 & 1 & & & & 0,493 & 2,885 & 0,687 \\
1994 & 0,868 & 1 & & & 0,494 & 2,717 & 0,737 \\
1995 & 0,859 & 0,900 & 1 & & 0,514 & 2,889 & 0,721 \\
1996 & 0,797 & 0,867 & 0,903 & 1 & 0,499 & 2,879 & 0,690 \\
\hline
\end{tabular}

Dans le Tableau II sont également indiqués les paramètres numériques suivants, appelés indices ou valeurs typologiques (CHESSEL et al., 1997) : (1) la variance vectorielle du tableau $k$, comme mesure de son inertie interne qui définit le degré de structure propre à chaque tableau et (2) le $\mathrm{Cos}^{2}$ entre le tableau $k$ et le compromis qui constitue un indicateur de la qualité de l'expression par un tableau donné de la structure compromis définie ici par les quatre premiers axes factoriels. La stabilité des valeurs des $\alpha_{k}$ et des variances vectorielles montrent que les participations de chacun des tableauxannées à la définition du compromis sont semblables ainsi que la structure des différents tableaux. 1995 se distingue cependant car elle correspond à l'année qui participe le plus à la construction du compromis et qui a la structure la plus forte (forte variance). Les valeurs élevées des $\operatorname{Cos}^{2}$ montrent que toutes les années sont bien représentées dans le compromis, 1994 étant la mieux représentée $\left(\operatorname{Cos}^{2}=0,737\right)$ et 1993 la moins bien $\left(\operatorname{Cos}^{2}=0,687\right)$.

\section{Analyse du compromis}

Les Figures $4 \mathrm{~B}$ et $4 \mathrm{C}$ représentent les positions " compromis " des espèces successivement dans les plans 1-2 et 3-4 et les Figures $5 A$ et $5 B$ celles des stations dans les mêmes plans.

L'axe 1 oppose la truite commune au chevaine. A proximité du centre du plan se trouvent les espèces "rares " peu prises en compte par l'ACP. Elles sont présentes dans seulement une ou deux stations et dans de très faibles proportions. II s'agit de l'épinoche, de l'ombre et du chabot. L'axe 2 oppose le barbeau fluviatile au barbeau méridional. Cette opposition reflète le comportement d'évitement du barbeau méridional vis-à-vis du barbeau fluviatile, espèce de plus grande taille et compétiteur intragénérique (BERREBI et al., 1988). Ce comportement se traduit par une séparation dans l'espace de leurs habitats respectifs. Le barbeau méridional est surtout présent dans des zones de semi-montagne dans les petits cours d'eau à faible débit pouvant être asséchés en été. Le barbeau 

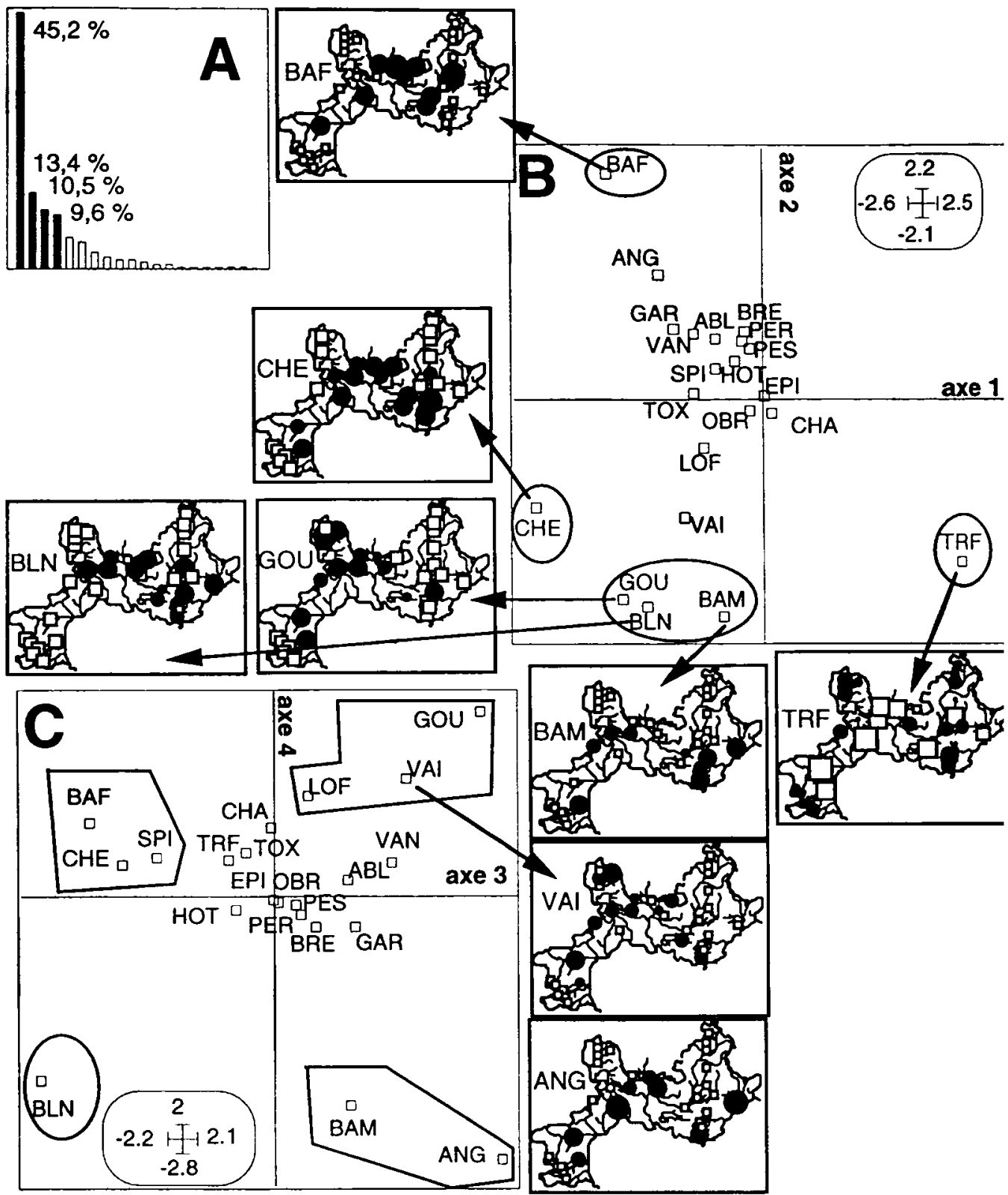

Figure 4

Analyse triadique partielle : analyse du compromis (espèces). (A) Histogramme des valeurs propres. (B) Projection des espèces sur le plan 1-2. (C) Projection des espèces sur le plan 3-4. En parallèle sont cartographiées les répartitions des espèces (valeurs issues du compromis). La surface des cercles (valeurs supérieures à la moyenne) et des carrés (valeurs inférieures à la moyenne) est proportionnelle aux données centrées.

\section{Figure 4}

Partial triadic analysis : compromise analysis (species). (A) Histogram of eigenvalues. (B) Projection of species taxa on the first two principal axes. (C) Projection of species taxa on the three-four principal axes. The species distributions are mapped (values calculated in the compromise). The surface of circles (values > mean) and squares (values < mean) is proportional to the centered values. 
fluviatile affectionne, lui, les cours d'eau de plaine. La compétition entre les deux espèces cantonne en effet le barbeau méridional dans les zones amont où les conditions du milieu sont trop contraignantes pour le barbeau fluviatile (CRESPIN et BERREBI, 1994). On le retrouve plus à l'aval uniquement dans les zones que le barbeau fluviatile n'a pu atteindre lors de la colonisation du bassin méditerranéen, probablement lors des dernières glaciations datant de 10 ou 15000 ans (PERSAT et BERREBI, 1990). La cartographie des biomasses centrées par espèce (Figure 4) montre la distribution de chacune des espèces dans les stations échantilionnées. On y observe effectivement que le barbeau méridional est absent des stations où le barbeau fluviatile est présent. Cette situation est nettement visible pour les cours d'eau côtiers du sud de la région PACA. Sur l'axe 2, le blageon et le goujon sont associés au barbeau méridional. Ces trois espèces ont des préférences d'habitat communes. Selon VERNEAUX (1981), le blageon occupe la zone s'étendant du mésorithron à l'épipotamon. CHANGEUX et PONT (1995) ajoutent qu'il se trouve très bien représenté dans les zones où séjournent les espèces les plus rhéophiles. Bien que pouvant se trouver aussi dans des grands cours d'eau de plaine (mésopotamon voire hypopotamon), le goujon partage avec le blageon les mêmes zones (du mésorithron à l'épipotamon). Entre ces deux pôles (barbeau méridional-barbeau fluviatile), les taxons se positionnent selon leur préférence d'habitat. En effet, du côté négatif de l'axe 2 (barbeau méridional), se trouvent des espèces d'eau vive comme le vairon ou la loche franche. Du côté positif de l'axe (barbeau fluviatile), se positionnent les espèces affectionnant les cours d'eau de plaine aux eaux faiblement courantes et plus chaudes : le gardon, l'ablette, la perche et la brème. L'anguille, migrateur thalassotoque, plus abondante dans les parties basses des cours d'eau côtiers, est associée à ce dernier groupe.

La projection des stations sur le plan 1-2 (Figure 5A), lue en parallèle avec celle des espèces sur le même plan (Figure 4A), permet d'identifier visuellement 3 groupes. Le premier (I) regroupe les stations dont le peuplement peut être qualifié de "peuplement à truite ". II est constitué de 11 stations dont le peuplement est composé uniquement de truites et de 4 stations où la truite est dominante et représente au minimum $87,5 \%$ de la biomasse (Aude (Aud), Sorgue de la Colonne (Sor), Têt (Tet) et Verdon (Ver)). Le deuxième groupe (II) correspond à des peuplements mixtes. On y trouve des stations pour lesquelles la truite représente généralement au moins $50 \%$ de la biomasse du peuplement total (Caramy (Car), Chapeauroux (Cha), Issole (Iss), Colagne (Cog), Gapeau (Gap) et Tes (Tes)). Elle est accompagnée de différentes espèces (barbeau méridional, blageon, goujon, loche franche, chevaine, ombre ou vairon), la composition spécifique du peuplement variant d'une station à l'autre. D'autres stations ont un peuplement dominé par une autre espèce que la truite. C'est le cas de l'Hérault (Her) dont le peuplement est dominé par le blageon, et du Loup (Lou) qui a la particularité d'avoir un peuplement caractéristique de certains cours d'eau côtiers méditerranéens avec la dominance de l'anguille ( $\geq 70 \%)$. Le groupe III est composé de stations où le peuplement est constitué essentiellement de cyprinidés avec une richesse spécifique plus importante que celle des deux groupes précédents (Annexe). On peut distinguer deux sous-groupes, les stations du premier (IIla) ont un peuplement dominé par le chevaine accompagné de barbeau méridional, de goujon ou de blageon (Bresque (Bre), Gardon de Saint Jean (Gar), Agly (Agl)) alors que le deuxième (IIlb) est plutôt caractérisé par un peuplement dominé par le chevaine ou le barbeau fluviatile (Arc (Arc), Asse (Ass), Sorgue Rode (Rod), Toulourenc (Tor), Réal de Jouques (Jou)). Parmi les stations du groupe IIlb, le Lez à Castelnau (LezC) a la particularité d'avoir un peuplement dominé par l'anguille dont l'importance peut s'expliquer par la proximité de la mer.

Le plan 3-4 des espèces (Figure 4C) met en opposition 4 groupes d'espèces : le groupe goujon, vairon, loche franche, espèces d'accompagnement de la truite, s'oppose selon l'axe 3, au blageon ainsi qu'au groupe barbeau fluviatile, chevaine, spirlin, cyprinidés dont la zone de prédilection est l'épipotamon (VERNEAUX, 1981) qui correspond à des cours d'eau de plaine, et selon l'axe 4 au groupe barbeau méridional-anguille. Ce groupe 


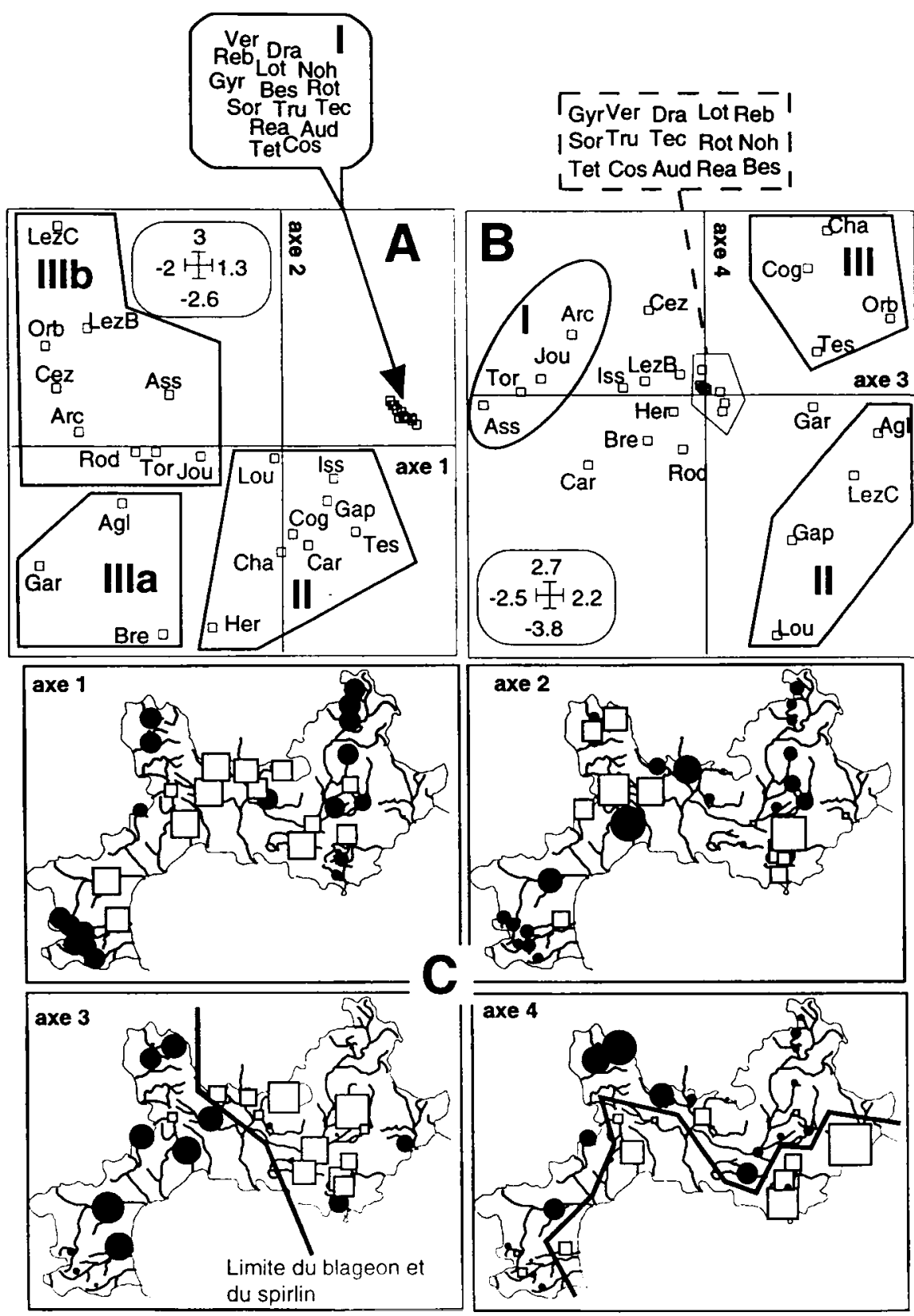

Figure 5

Analyse triadique partielle : analyse du compromis (stations). (A) Projection sur le plan 1-2. (B) Projection sur le plan 3-4. (C) Cartographie des coordonnées des stations sur les quatre premiers axes du compromis. Les cercles représentent les valeurs positives et les carrés représentent les valeurs négatives, les surfaces des cercles et des carrés sont proportionnelles à ces valeurs.

Figure 5

Partial triadic analysis : compromise analysis (stations). (A) Projection on the first two principal axes. (B) Projection on the three-four principal axes. (C) Stations scores mapping in reference to the first four axes of the compromise. Circles represent positive values and squares represent negative values, the surfaces of circles and squares are proportional to these values. 
s'oppose lui-même au blageon selon l'axe 3. Ces trois dernières espèces sont inféodées soit à la région méditerranéenne ou aux Alpes du Sud (barbeau méridional, blageon), soit à des cours d'eau côtiers (anguille). En effet, la distribution du blageon est centrée sur les Alpes et sa limite de répartition se situe à l'ouest au fleuve Hérault (BANARESCU, 1990 ; CHANGEUX et PONT, 1995). Originaire du nord de l'Europe, le spirlin est localisé dans la moitié Est du pays, le département de l'Hérault constituant également sa limite de répartition à l'Ouest (ALLARDI et KEITH, 1991 ; BILLARD, 1997). Ainsi, en regardant les cartographies des coordonnées des stations sur les axes 3 et 4 du compromis (Figure 5C), il apparaît clairement que ce plan met en évidence d'une part un effet régionalisation EstOuest par l'axe 3 qui s'explique en partie par les distributions du blageon et du spirlin, et un effet régionalisation Nord-Sud (cours d'eau côtiers ou de plaine-cours d'eau d'altitude) par l'axe 4. Plus précisément, sur le plan 3-4 (Figure 5B), se distinguent trois groupes de stations: (I) les stations de l'Est sud-est contenant majoritairement du barbeau fluviatile, du blageon et du chevaine, associés ou non à du spirlin (Arc (Arc), Asse (Ass), Réal de Jouques (Jou), Toulourenc (Tor)) ; (II) les stations de cours d'eau côtiers dont les peuplements sont typiques de la région méditerranéenne : anguille, barbeau méridional et blageon pour les cours d'eau situés à l'est (Gapeau (Gap), Loup (Lou)) ; (III) les stations situées uniquement en région LR parmi lesquelles la Colagne (Cog) et le Chapeauroux (Cha) dont les peuplements se rapprochent de ceux des cours d'eau du Massif central (peuplements mixtes).

Ainsi, le plan 3-4, représentant $20 \%$ de l'inertie, met en évidence une forte structuration régionale.

\section{Analyse de l'évolution temporelle autour de la structure du compromis}

La reproductibilité de la structure du compromis dans le temps peut être représentée pour les espèces et les stations et permet de discuter de l'évolution temporelle des relations espèces-stations (typologie interne de chaque tableau) par projection des lignes et des colonnes de chacun des tableaux sur les plans définis par le compromis.

La projection des axes principaux 1-2 et 3-4 des analyses séparées par date, respectivement sur le plan 1-2 (Figure 6A) et 3-4 (Figure 7A) du compromis, fournit des représentations de la contribution de chaque tableau-date à la formation du compromis. Elle montre que les axes 1 et 2 et 3 et 4 de chacune des analyses séparées se superposent quasiment (à une rotation près) à ceux du compromis. Cette observation concorde bien avec les résultats fournis par les valeurs des indices typologiques (Tableau II).

Les Figures $6 \mathrm{~B}$ et $6 \mathrm{C}$ (plan 1-2) mettent effectivement en évidence une stabilité importante dans la répartition des espèces ou des stations entre 1993 et 1996. En effet, même si les positions des espèces ou des stations varient d'une année à l'autre, elles restent dans le même " secteur " du plan du compromis. Cependant, par rapport à l'image fournie par le compromis (Figure 4), on note une décroissance de l'importance de l'anguille dans la définition de l'axe 2 si bien qu'en 1996, elle n'apparaît plus comme discriminante. On observe effectivement une diminution continue des populations de cette espèce dans les deux stations où elle domine le peuplement (le Loup et le Lez à Castelnau). Cette réduction des populations d'anguilles semble être un phénomène général à l'ensemble de l'Hexagone (CHANCEREL, 1994). Les causes sont multiples : surexploitation des juvéniles, influences anthropiques augmentant les difficultés à atteindre les zones de grossissement, effet du parasite Anguillicola crassus... et il est difficile de fournir une explication unique à ce phénomène. Plus ou moins fluctuante entre 1993 et 1995, la position du gardon semble au contraire se rapprocher de celle du barbeau fluviatile en 1996, participant ainsi à la définition de l'axe 2. Ceci peut être lié à l'acclimatation de cette espèce sur la station de l'Agly suite à des déversements dans un but halieutique. 


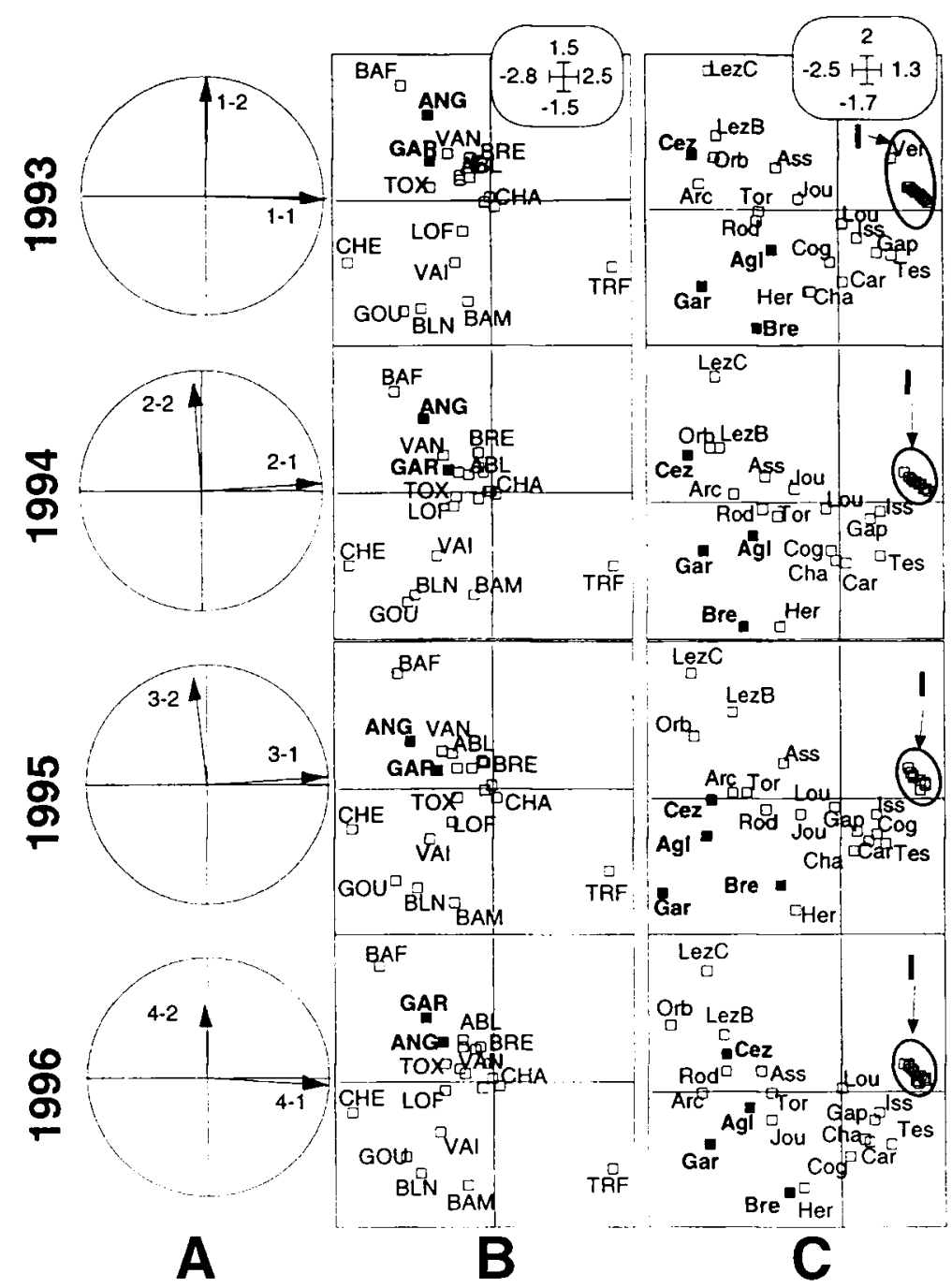

Figure 6

Analyse triadique partielle : analyse de l'évolution temporelle autour de la structure compromis (plan 1-2). (A) Projection des axes principaux issus des ACP séparées sur le plan 1-2 du compromis. Le premier caractère identifie le numéro du tableau, le second représente le numéro de l'axe de l'analyse séparée. (B) Projection sur le plan 1-2 du compromis des coordonnées des colonnes (espèces) des ACP séparées. (C) Projection sur le plan 1-2 du compromis des coordonnées des lignes (stations) des ACP séparées, I correspond aux stations à truite identifiées par le compromis (cf. Figure 5A).

\section{Figure 6}

Partial triadic analysis : analysis of temporal variation around the compromise structure (1-2 principal axes). (A) Projection of the first two principal axes of the separate PCAs on the 1-2 principal axes of the compromise. The first character identifies the table number and the second represents the axis number of the corresponding separate analysis. (B) Projection of the species scores produces by the separate PCAs on the 1-2 principal axes of the compromise. (C) Projection of the stations scores produces by the separate PCAs on the 1-2 principal axes of the compromise, I corresponds to the trout stations identified by the compromise (see Figure 5A). 
On note également une stabilité importante dans la position des stations entre 1993 et 1996. Suivant les années, certaines stations semblent tout de même changer de position. C'est par exemple le cas, en 1995, de l'Agly (Agl) et du Gardon de St Jean (Gar). On observe cette année-là sur ces stations une augmentation significative de la biomasse du chevaine, du goujon, du barbeau méridional, du blageon et du gardon si bien que, par rapport aux autres années, la biomasse totale fait plus que doubler sur l'Agly et triple sur le Gardon de Saint Jean. Ces augmentations pourraient être liées au régime hydrologique, avec des débits plus faibles en 1995. Ces fortes valeurs de biomasse font que ces deux stations sont mieux représentées dans le plan cette année-là. Par rapport à l'image de référence fournie par le compromis, il y a peu de différence. Ceci confirme les informations données par les valeurs élevées des indices typologiques (Tableau II) qui montrent que 1995 est l'année qui participe le plus à la construction du compromis (poids $\alpha_{k}$ le plus important) et qui, de plus, est bien représentée par celui-ci (fort $\operatorname{Cos}^{2}$ ). 1995 semble être par contre une année atypique par rapport à l'image de référence pour la station située sur la Cèze ( $\mathrm{Cez}$ ). On constate en effet une augmentation des proportions des petites espèces : goujon, loche franche, vairon et blageon. Des travaux destinés à canaliser le débit pour faciliter la pratique du canoë ont entrainé une régression des faciès lotiques, des profondeurs moyennes et rendu inaccessibles les principales caches. Ces modifications de l'habitat ont entraîné une réduction du nombre d'adultes notamment pour le barbeau fluviatile, le chevaine, le toxostome et le gardon et, par suite, une diminution de la biomasse globale d'où une moins bonne représentation dans le plan de cette station. Mais les crues d'automne ont rendu à la station sa morphologie initiale et le peuplement observé en 1996 est de nouveau proche de celui de 1993 et 1994. Enfin, la position de la Bresque (Bre), dont le peuplement est un peu atypique (dominance du chevaine et présence simultanée du barbeau méridional, du blageon, du goujon et de la truite), évolue suivant les années. Se situant plutôt avec les stations à cyprinidés en 1993 et 1994, sa position évolue vers les stations à peuplement mixte à partir de 1995. Ceci peut s'expliquer par la diminution du chevaine suite au déplacement vers l'aval en 1995 de l'exutoire de la station d'épuration de Salernes. L'importance de cette espèce les années précédentes semble, en effet, être liée aux rejets.

Les Figures 7B et 7C (plan 3-4) mettent également en évidence une stabilité importante de la répartition des espèces et des stations entre 1993 et 1996 . Cependant, on observe, par rapport à l'image fournie par le compromis (Figure $4 \mathrm{C}$ ), une évolution de la position de la truite et du chevaine. En 1993, la truite est située du côté positif de l'axe 3 alors qu'elle est positionnée du côté négatif les trois années suivantes. II ne semble pas y avoir d'explication écologique à cette évolution mais nous pouvons supposer que ceci pourrait être lié au fait que l'année 1993 est la moins bien représentée dans le compromis (Tableau II : valeurs de $\alpha_{k}$ et de $\operatorname{Cos}^{2}$ les plus faibles). De plus, la mauvaise représentation de cette espèce dans le plan relativise l'importance des variations de sa position dans le plan.

L'analyse de l'évolution de la position des stations sur le plan 3-4 montre aussi des changements d'une année sur l'autre. Le Réal de Jouques (Jou) a en 1993 une position très différente de celle du compromis et des années suivantes. La présence cette année-là de l'association d'espèces loche franche-vairon explique sa position du côté positif de l'axe 3 . Cette association ne se retrouvant pas les années suivantes (seule la loche franche est capturée à nouveau en 1996), la station évolue vers le côté négatif de l'axe 3. Les fluctuations de la position de cette station dans le plan peuvent s'expliquer par les variations d'une année sur l'autre de sa richesse spécifique (oscillant entre 4 et 8 , cf. Annexe) et de la structure de son peuplement en fonction des influences anthropiques (déversements, présence d'une station d'épuration en amont) et du régime hydrologique (crues). La position du Gardon de Saint Jean (Gar) fluctue également d'une année à l'autre, celle de 1995 étant la plus proche de celle observée dans l'analyse du compromis. Alors qu'en 1993 l'anguille est absente du peuplement, sa présence les années suivantes peut expliquer en partie le 


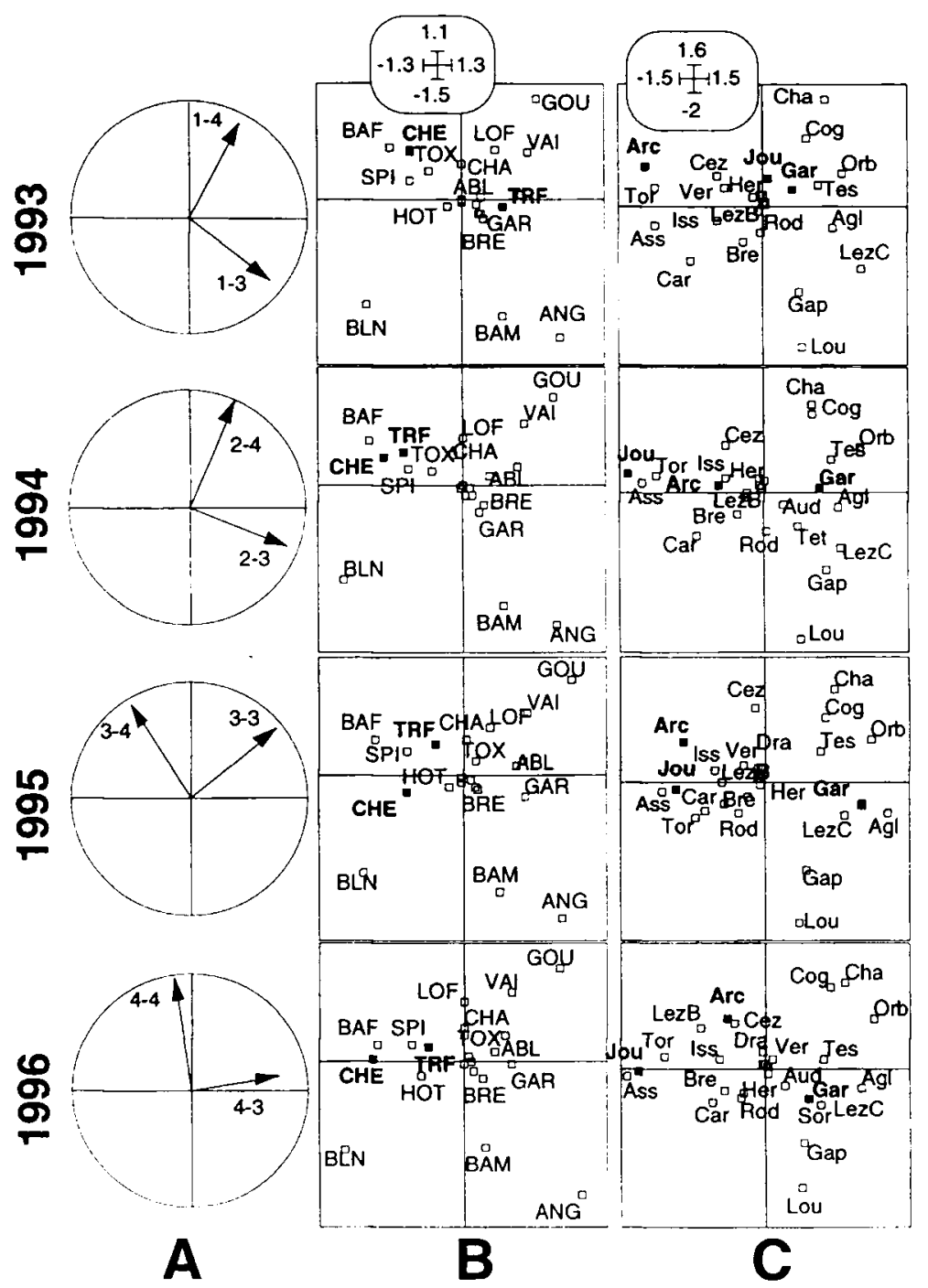

Figure 7

Analyse triadique partielle : analyse de l'évolution temporelle autour de la structure compromis (plan 3-4). (A) Projection des axes principaux issus des ACP séparées sur le plan 3-4 du compromis. Le premier caractère identifie le numéro du tableau, le second représente le numéro de l'axe de l'analyse séparée. (B) Projection sur le plan 3-4 du compromis des coordonnées des colonnes (espèces) des ACP séparées. (C) Projection sur le plan 3-4 du compromis des coordonnées des lignes (stations) des ACP séparées.

\section{Figure 7}

Partial triadic analysis : analysis of temporal variation around the compromise structure (3-4 principal axes). (A) Projection of the first two principal axes of the separate PCAs on the 3-4 principal axes of the compromise. The first character identifies the table number and the second represents the axis number of the corresponding separate analysis. (B) Projection of the species scores produces by the separate PCAs on the 3-4 principal axes of the compromise. (C) Projection of the stations scores produces by the separate PCAs on the 3-4 principal axes of the compromise. 
déplacement de la position de la station sur le plan 3-4. Concernant l'Arc, la position observée durant l'été 1994 est la plus éloignée du compromis. Cette variation traduit l'influence des crues de janvier et février 1994 qui ont réduit le peuplement en place et modifié la proportion des différentes espèces. En 1995, le peuplement observé initialement se reconstitue partiellement mais reste limité par la modification des habitats. La position de l'Arc se rapproche alors de celle du compromis.

Ainsi, l'analyse triadique partielle permet de montrer qu'il existe pour les peuplements faunistiques des régions Languedoc-Roussillon et Provence-Alpes-Côte d'Azur une structure spatiale espèces-stations (structure compromis) qui est stable sur les quatre années étudiées.

\section{AFC de FOUCART sur les quatre tableaux faunistiques}

L'analyse triadique partielle, utilisée ci-dessus pour identifier une structure spatiale de référence fonctionne avec une logique d'ACP. L'accent est donc mis sur les variations d'abondance des espèces qui peuvent être liées à des perturbations locales (pollution, hydrologie...). En choisissant une stratégie d'AFC, dont le double centrage élimine les variations d'abondance (DOLÉDEC et CHESSEL, 1991), on élimine les effets de ces perturbations. Du fait de la symétrie inhérente à la méthode, on s'intéresse à la fois aux profils de répartition des espèces entre les stations et symétriquement, aux profils de répartition des stations entre les espèces.

L'AFC de FOUCART sur les quatre tableaux-dates faunistiques calcule un tableau compromis moyen qui s'analyse comme une AFC classique. La distribution des espèces dans le plan 1-2 du compromis de l'AFC de FOUCART (Figure 8B) rappelle celle des schémas classiques de zonation (HUET, 1954 ; ILLIES et BOTOSANEANU, 1963) ou de typologie (VERNEAUX, 1973, 1981). On y retrouve successivement la zone à truite (truite, chabot), la zone à ombre, celle à barbeau puis celle à brème. Toutefois, par rapport à ce schéma on note des nuances remarquables. Par rapport à la typologie de VERNEAUX, on observe que la truite est la première espèce à apparaître dans la zonation piscicole. L'importance moindre du chabot est liée à son absence d'un certain nombre de têtes de bassin dans lesquelles seule la truite est présente (notamment ceux des PyrénéesOrientales, certains côtiers est...). On ne peut pas tirer de conclusion sur la position de l'épinoche au côté de la truite. II s'agit en effet d'une espèce rare, présente uniquement sur deux stations du bassin des Sorgues, qui colonise généralement des milieux marginaux (petits ruisseaux ou fossés en bordure de cours d'eau, zones peu profondes pourvues de végétation aquatique) indépendamment de tout preferendum au sein du continuum amontaval. Le barbeau méridional, conformément à la classification établie par HUET (1954), se trouve avec les espèces de la zone à ombre (vairon, blageon, ombre), ce qui se justifie par leur préférence commune pour les eaux vives. Ajoutons ici que la dénomination de zone à ombre n'est d'ailleurs pas la plus adaptée aux régions concernées, étant donné la faible occurrence de cette espèce. Or, le blageon, plus répandu, est considéré comme l'espèce indicatrice remplaçant l'ombre dans les rivières du sud (LEGER, 1937). Le terme de "zone à blageon " nous semble donc plus approprié. Située entre la zone à barbeau et la zone à brème, l'anguille est légèrement isolée. Du fait de sa présence dans des stations aux peuplements différents et de son fort caractère migrateur, il est difficile de la rattacher à une zone précise ou de lui attribuer un preferendum typologique. La loche franche, connue comme espèce d'accompagnement de la truite, se positionne ici à la limite de la zone à ombre et de la zone à barbeau. Ceci correspond à sa présence dans des cours d'eau à la richesse spécifique relativement élevée dans lesquels la truite est faiblement représentée alors que le chevaine, le goujon ou le barbeau le sont fortement. L'association de la vandoise avec les espèces de la zone à brème n'est pas significative. Elle reflète la très faible occurrence de cette espèce, capturée uniquement sur 4 stations et le faible nombre de stations à cyprinidés d'eaux calmes (zone à brème) prises en compte dans l'analyse. 


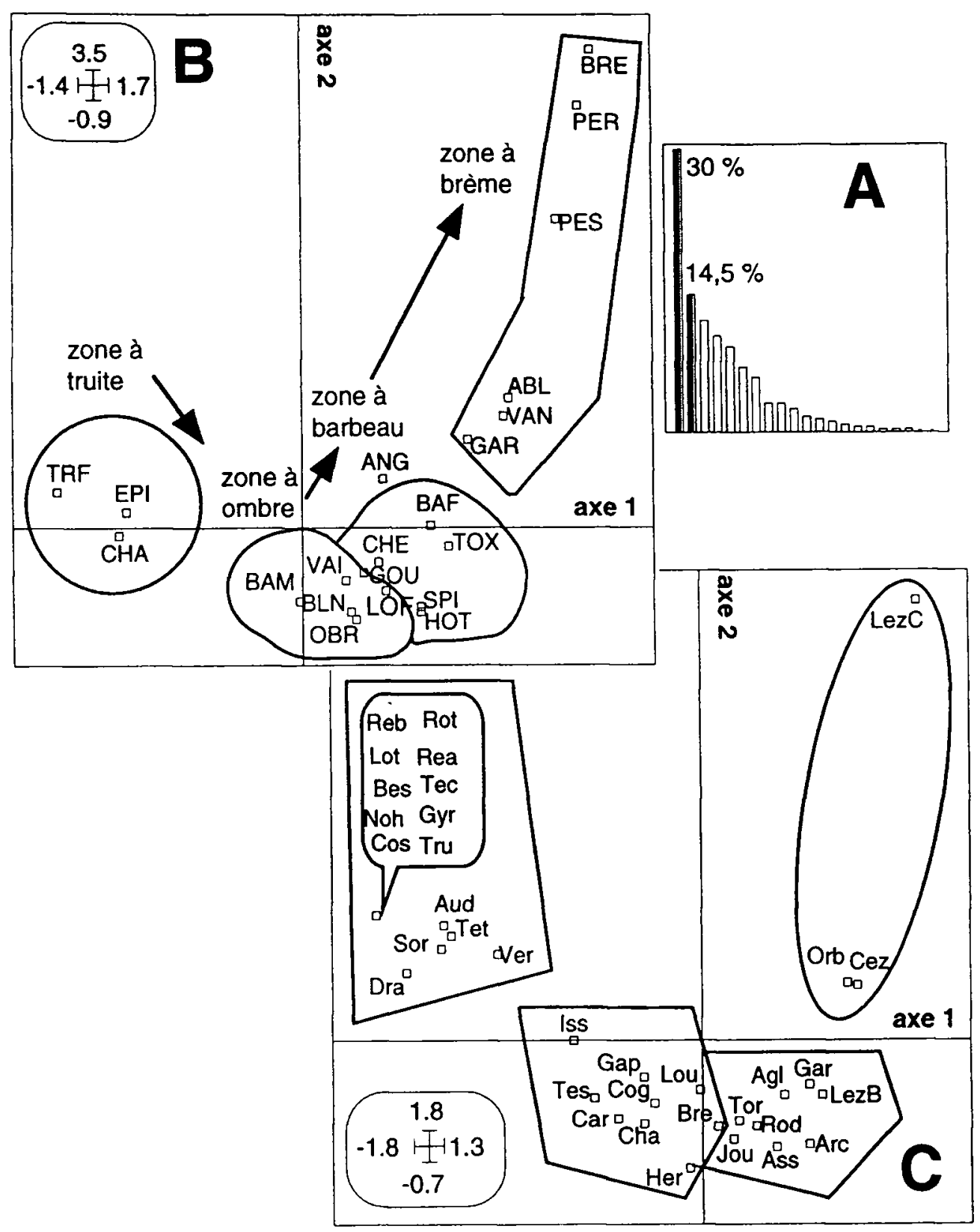

Figure 8

AFC de FOUCART : analyse de la structure compromis. (A) Histogramme des valeurs propres. (B) Projection des espèces sur le plan 1-2 du compromis. (C) Projection des espèces sur le plan 3-4 du compromis.

Figure 8

FOUCART's correspondence analysis : analysis of the compromise structure. (A) Histogram of eigenvalues. (B) Projection of species taxa on the first two principal axes. (C) Projection of species taxa on the three-four principal axes. 


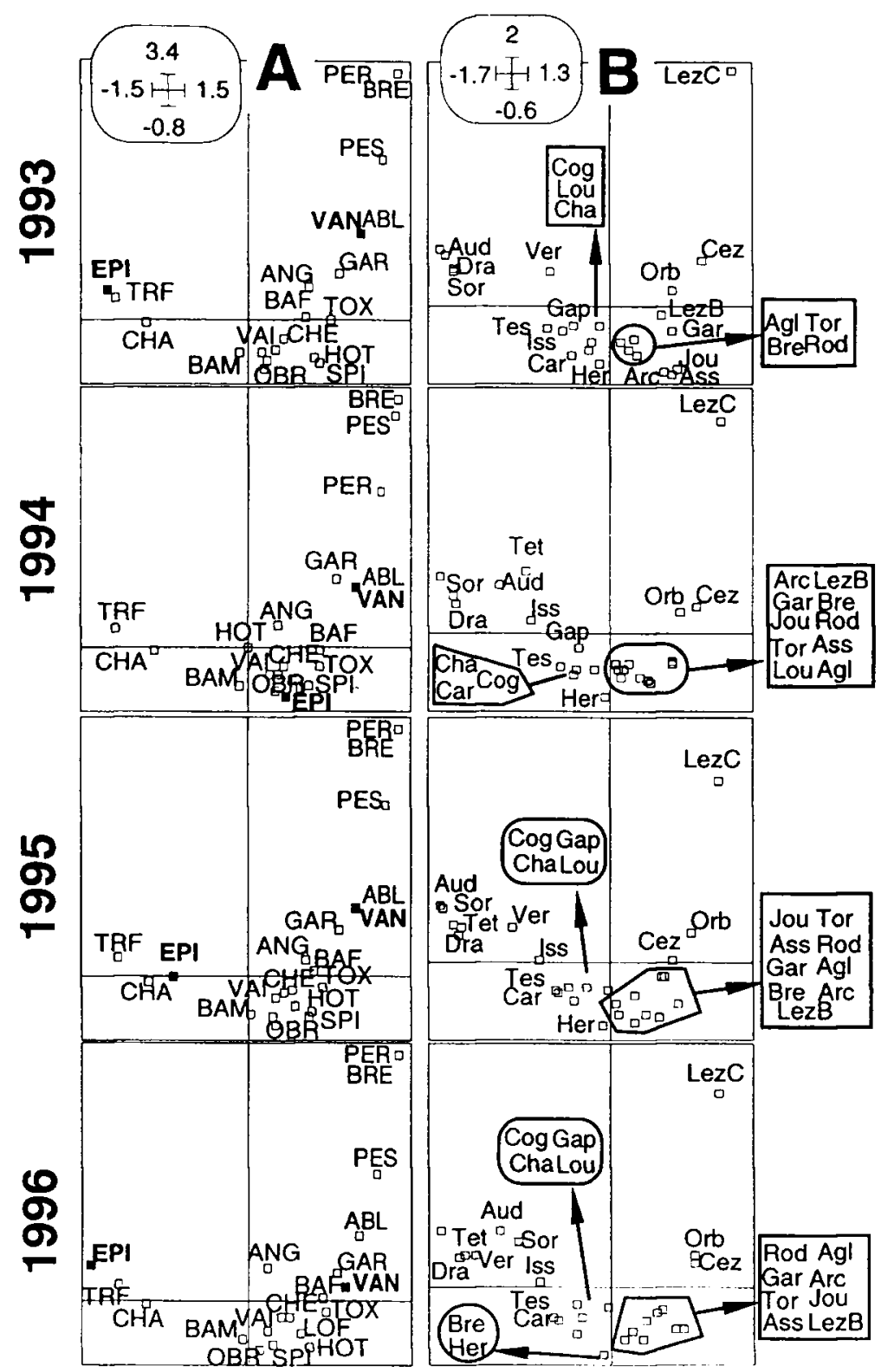

Figure 9

AFC de FOUCART : analyse de l'évolution temporelle autour de la structure compromis. (A) Projection des colonnes (espèces) de chaque tableau (année) sur le plan 1-2 de la structure compromis. (B) Projection des lignes (stations) de chaque tableau (date) sur le plan 1-2 de la structure compromis. Les codes des stations situées dans la zone à truite (voir Figure 8) et qui ne bougent pas d'une année sur l'autre ne figurent pas sur ces plans.

\section{Figure 9}

FOUCART's correspondence analysis : analysis of temporal variation around the compromise structure. (A) Projection of columns (species) of each table (year) on the first factorial plane of the compromise structure. (B) Projection of rows (stations) of each table (year) on the first factorial plane of the compromise structure. The codes of stations which are in the trout zone (see Figure 8) and which do not move from year to another are not represented on these planes. 
Le plan des stations (Figure $8 \mathrm{C}$ ) montre des regroupements très proches de ceux que nous avions identifiés avec l'analyse triadique partielle : les stations de la zone à truite stricto sensu, celles de la zone à ombre que nous avions qualifiées de stations à peuplement mixte et celles de la zone à barbeau que nous avions appelées stations à cyprinidés. Trois stations se distinguent nettement : le Lez à Castelnau (LezC), l'Orbieu (Orb) et la Cèze (Cez). Ces deux dernières, situées à la limite de la zone à barbeau et de la zone à brème ont un peuplement caractérisé par la présence simultanée de l'ablette et de la vandoise. Le Lez à Castelnau est la seule station échantillonnée dans laquelle se trouvent les trois espèces " repères " (brème, perche et perche soleil), ce qui explique la position extrême de cette station dans le plan.

La projection en individus supplémentaires des lignes et des colonnes de chacun des tableaux initiaux dans le plan du compromis (Figures 9A et 9B) montre une certaine stabilité de la structure identifiée par le compromis. L'évolution des positions des espèces rares (épinoche, vandoise) est peu significative, puisqu'essentiellement liée à leurs fluctuations d'occurrence suivant les années. Les positions de certaines stations situées dans la zone à truite (Figure 9B) semblent évoluer selon les années mais cependant elles ne quittent pas cette zone. II s'agit des stations où une faible proportion d'espèces d'accompagnement sont présentes. Leurs variations d'abondance se traduisent par une légère modification de la position des stations dans le plan compromis. La limite entre la zone à ombre et celle à barbeau n'étant pas bien définie, les stations correspondant à cette localisation charnière peuvent passer d'une zone à l'autre mais, globalement, les variations d'une année sur l'autre ne semblent pas significatives.

\section{CONCLUSION}

Deux méthodes d'analyse multitableaux sont utilisées pour étudier les peuplements piscicoles des régions Languedoc-Roussillon et Provence-Alpes-Côte d'Azur à partir des données issues du Réseau Hydrobiologique et Piscicole. Elles permettent d'identifier une structure spatiale espèces-stations commune à toutes les années.

Dans un premier temps, l'analyse triadique partielle met en évidence d'une part une opposition entre des stations à truite ou à peuplement mixte et des stations à cyprinidés d'eau vive. D'autre part, un double effet " région " conditionne cette typologie spatiale : une régionalisation Est-Ouest qui s'explique pour une large part par les distributions géographiques du blageon et du spirlin et une régionalisation Nord-Sud isolant les cours d'eau côtiers de la région méditerranéenne qui est liée à la présence de deux espèces inféodées à cette région, l'anguille et le barbeau méridional. Même si l'étude de l'évolution temporelle autour de cette structure moyenne montre une forte stabilité de la structure spatiale espèces-stations entre 1993 et 1996, des fluctuations ponctuelles ont pu être observées. Elles sont généralement dues à des perturbations d'origine anthropique ou climatiques avec notamment de fortes crues ou des étiages sévères.

Dans un second temps, l'AFC moyenne de FOUCART identifie par le compromis une distribution des espèces sur un continuum amont-aval proche de celle des schémas classiques de zonation (HUET, 1954 ; ILLIES et BOTOSANEANU, 1963) ou de typologie (VERNEAUX, 1973, 1981). Néanmoins, des différences existent par rapport à ces schémas. Elles s'expliquent principalement par l'existence de stations situées dans les têtes de bassin dans lesquelles on ne trouve que de la truite, par la présence du barbeau méridional et par la biomasse importante de l'anguille dans certains cours d'eau. De plus, cette structure identifiée par le compromis se révèle également stable sur la période d'étude. 
La mise en oeuvre de ces deux méthodes nous permet d'obtenir des informations complémentaires et détaillées sur les structures spatiales espèces-stations et sur leur stabilité temporelle. A la différence de l'analyse triadique partielle qui met en évidence un fort effet région, I'AFC moyenne de FOUCART permet d'identifier une distribution des espèces sur un continuum amont-aval indépendamment des effets régionaux. De plus, l'analyse triadique partielle, fondée sur la logique de I'ACP, prend en compte les fluctuations d'abondance et souligne donc les perturbations éventuelles subies par le milieu. L'AFC de FOUCART, fondée sur la logique de l'AFC, met en évidence des associations d'espèces indépendamment de leur abondance. Elle valorise donc le rôle des espèces rares.

Ainsi, aux deux questions posées initialement, à savoir : (1) peut-on mettre en évidence une structure spatiale ? (2) Cette structure est-elle stable temporellement ? Nous répondons par l'affirmative. Cette stabilité temporelle de la structure spatiale souligne l'existence d'une structuration typologique des peuplements piscicoles des régions Languedoc-Roussillon et Provence-Alpes-Côte d'Azur qui incluent la région méditerranéenne. II faut cependant souligner que dans la présente étude tous les types de peuplement ne sont pas représentés (absence des zones potamiques). Dans la continuité de ce travail et pour aller plus loin dans l'étude de l'écologie des communautés, il

\section{Annexe}

Taux d'occurrence des espèces dans chaque station calculés sur les quatre années et richesse spécifique : valeur minimale-valeur maximale sur les quatre années. Les données brutes sont disponibles auprès des auteurs.

\section{Appendix}

Occurrency rates of species in each station calculated for the four years and species richness : minimal value and maximal value over the four years. Raw data are available near authors.

\begin{tabular}{|c|c|c|c|c|c|c|c|c|c|c|c|c|c|c|c|c|c|c|c|c|c|c|}
\hline \multirow[t]{2}{*}{ ST } & \multicolumn{21}{|c|}{ TAUX D'OCCURRENCE } & \multirow[t]{2}{*}{ AS } \\
\hline & $A B L$ & ANG & BAF & BAM & BLN & BRE & $\mathrm{CHA}$ & $\mathrm{CHE}$ & EPI & GAP & GOU & HOI & LOF & $\mathrm{OBR}$ & PER & PES & SPI & Tox & TRF & VAI & VAN & \\
\hline Oro & 1 & 1 & 1 & 0 & 0 & 0 & 0 & 1 & 0 & 0.5 & 1 & 0 & 1 & 0 & 0 & 0 & 0 & 0.5 & 0.5 & 1 & 1 & $9 \cdot 10$ \\
\hline Reb & 0 & 0 & 0 & 0 & 0 & 0 & 0 & 0 & 0 & 0 & 0 & 0 & 0 & 0 & 0 & 0 & 0 & 0 & 1 & 0 & 0 & 1 \\
\hline Gar & 1 & 0.75 & 0.25 & 1 & 1 & 0 & 0.25 & 1 & 0 & 0.75 & 1 & 0 & 1 & 0.25 & 0 & 0 & 0 & 1 & 1 & 1 & 1 & $11 \cdot 13$ \\
\hline Her & 0 & 0 & 0 & 1 & 1 & 0 & 0 & 1 & 0 & 0 & 1 & 0 & 0 & 0 & 0 & 0 & 0 & 0 & 1 & 1 & 0 & 6 \\
\hline Cez & 1 & 0 & 1 & 0 & 1 & 0 & 0 & 1 & 0 & 1 & 1 & 0 & 1 & 0 & 0.25 & 1 & 1 & 1 & 0.25 & 1 & 1 & $12 \cdot 13$ \\
\hline Lot & 0 & 0 & 0 & 0 & 0 & 0 & 0 & 0 & 0 & 0 & 0 & 0 & 0 & 0 & 0 & 0 & 0 & 0 & ' & 0 & 0 & 1 \\
\hline $\mathrm{Cog}$ & 0 & o & 0.25 & 0 & 0 & 0 & 0.25 & 0.75 & 0 & 0 & 1 & 0 & 1 & 0 & 0 & 0 & 0 & 0 & 1 & , & 0 & 4.5 \\
\hline Cha & 0 & 0 & 0 & 0 & 0 & 0 & 1 & 1 & 0 & 0 & 1 & 0 & 1 & 0.75 & 0 & 0 & 0 & 0 & 1 & 1 & 0 & 6.7 \\
\hline Tru & c & 0 & 0 & 0 & 0 & 0 & 0 & 0 & 0 & 0 & 0 & 0 & 0 & 0 & 0 & 0 & 0 & 0 & 1 & 0 & 0 & 1 \\
\hline Agl & 0.25 & 9 & 0 & : & 0 & 0 & 0 & $i$ & 0 & 1 & 1 & 0 & 1 & 0 & 0 & 0 & 0 & 0 & 0.25 & 1 & 0 & 7.8 \\
\hline Rot & 0 & 0 & 0 & 0 & 0 & 0 & 0 & 0 & 0 & 0 & 0 & 0 & 0 & 0 & 0 & 0 & 0 & 0 & 1 & 0 & 0 & 1 \\
\hline $\cos$ & 0 & 2 & 0 & 0 & 0 & 0 & 0 & 0 & 0 & 0 & 0 & 0 & 0 & 0 & 0 & 0 & 0 & 0 & 1 & 0 & 0 & 1 \\
\hline Bes & 0 & 0 & 0 & 0 & 0 & 0 & 0 & 0 & 0 & 0 & 0 & 0 & 0 & 0 & 0 & 0 & 0 & 0 & 1 & 0 & 0 & 1 \\
\hline Ver & 0 & 0 & 0.5 & 0 & 0 & 0 & 0 & 0 & 0 & 0 & 0 & 0 & 0 & 0 & c & 0 & 0 & 0 & 1 & 0.5 & 0 & 1.3 \\
\hline Gyr & 0 & 0 & 0 & 0 & 0 & 0 & 0 & 0 & 0 & 0 & 0 & 0 & 0 & 0 & $\hat{\approx}$ & 0 & 0 & 0 & 1 & 0 & 0 & 1 \\
\hline Rea & 0 & 3 & $c$ & 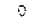 & 0 & 5 & 0 & 0 & 0 & 0 & 0 & 0 & c & 0 & 3 & 0 & 0 & 0 & 1 & 0 & $c$ & 1 \\
\hline Dra & 0 & 2 & 0 & 0 & c & 0 & 1 & c & 0 & 0 & 0 & 0 & 0 & 0 & $\therefore$ & 0 & 0 & 0 & $\cdot$ & 0 & 3 & 2 \\
\hline Lou & c & 1 & 0 & $\cdot$ & i & 0 & 0 & 0 & 0 & 0 & a & 0 & 0 & c & $=$ & 0 & 0 & 0 & ! & 0 & 0 & 4 \\
\hline Jou & c & 0 & 0.75 & 0.5 & 0.75 & 0 & 0 & 1 & 0 & 0.25 & 1 & 0 & 0.25 & c & $\hat{\imath}$ & 0 & 1 & 0 & 0,75 & 0 & 0 & 4.8 \\
\hline AiC & 0 & 0.25 & 1 & 0 & 1 & 0 & 0 & 1 & 0 & 0.5 & 1 & 0 & 1 & 0 & 0 & 0.25 & 1 & 0.25 & 0.75 & 0.75 & 0 & 8.9 \\
\hline Gap & 0 & 1 & c & 1 & $i$ & 0 & 2 & 0 & 0 & 0 & 3 & 0 & 0 & 0 & 3 & 0 & 0 & 0 & 1 & 1 & 0 & 4.5 \\
\hline Car & 0 & 0 & c & $:$ & $:$ & 0 & 0 & i & 0 & 0 & 0 & 0 & 0 & 0 & 2 & 0 & 0 & 0 & 1 & 0 & 0 & 4 \\
\hline Bre & 0 & 0 & 0 & 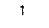 & $!$ & 3 & 0 & 1 & 0 & 0.75 & : & 0 & 0 & 0 & 0 & 0 & 0 & 0 & 1 & 0.5 & 0 & $5 \cdot 7$ \\
\hline
\end{tabular}


conviendrait de prendre également en compte des descripteurs environnementaux des stations échantillonnées comme l'altitude, la distance à la source, la pente, la qualité physico-chimique de l'eau... A partir de l'étude des relations entre la structure spatiale basée sur le peuplement piscicole des stations et celle basée sur les paramètres du milieu, une co-structure entre relevés faunistiques et relevés mésologiques pourrait alors être mise en évidence.

\section{REMERCIEMENTS}

Ce travail a été réalisé dans le cadre d'une convention d'étude financée par le Conseil Supérieur de la Pêche et établie entre cet organisme, le C.N.R.S. et le Laboratoire d'Hydrobiologie Marine et Continentale de l'Université Montpellier II dirigé par Monsieur le Professeur G. LASSERRE. Nous remercions Messieurs E. VIGNEUX, responsable du Service de l'Informatique et des Relations Scientifiques du CSP, G. LASSERRE sans lesquels l'élaboration de cette convention n'aurait pas eu lieu ainsi que les membres de la Délégation Régionale $n^{\circ} 8$ du CSP et en particulier Monsieur J. MIELOT, Délégué régional, pour son accueil dans ses locaux et son soutien actif à ce travail et enfin Madame C. ALIAUME-ZERBI, maître de conférence à l'Université Montpellier II, pour la lecture attentive de ce manuscrit et ses critiques constructives. Certaines stations prises en compte dans ce travail ont été échantillonnées dans le cadre de conventions d'étude entre l'Agence de l'Eau Rhône-Méditerranée-Corse et la DR 8 du CSP.

\section{BIBLIOGRAPHIE}

ALLARDI J., KEITH P., 1991. Atlas préliminaire des poissons d'eau douce de France. Muséum National d'Histoire Naturelle de Paris / Conseil Supérieur de la Pêche / Centre National du Machinisme Agricole du Génie Rural, des Eaux et des Forêts / Ministère de l'Environnement, Paris, $232 \mathrm{p}$.

BANARESCU P., 1990. General distribution and dispersal of freshwater animals. In BANARESCU P., Zoogeography of freshwaters, $\mathrm{n}^{\circ} 1,511$ p., Aula-Verlag, Wiesbaden.

BERREBI P., LAMY G., CATTANEO-BERREBI G., RENNO J.F., 1988. Variabilité génétique de Barbus meridionalis risso (Cyprinidae) : une espèce quasi monomorphe. Bull. Fr. Pêche Piscic., 310, 77-84.

BILLARD R., 1997. Les poissons d'eau douce des rivières de France. Delachaud et Niestlé S.A., Lausanne, Paris, 192 p.

BLANC L., CHESSEL D., DOLÉDEC S., 1998. Étude de la stabilité temporelle des structures spatiales par analyses d'une série de tableaux de relevés faunistiques totalement appariés. Bull. Fr. Pêche Piscic., 348.

BOËT P., ALLARDI J., LEROY J., 1991. Le peuplement ichtyologique du bassin de I'Yonne. Bull. Fr. Pêche Piscic., 320, 7-28.

CARREL G., PONT D., RIVIER B., 1995. Variabilité temporelle des peuplements piscicoles dans la section médiane du Bas-Rhône. Bull. Fr. Pêche Piscic., 337/338/339, 101 111.

CHANCEREL F., 1994. Note technique - La répartition de l'Anguille en France. Bull. Fr. Pêche Piscic., 335, 289-294.

CHANGEUX T., 1995. Structure du peuplement piscicole à l'échelle d'un grand bassin européen : organisation longitudinale, influence de la pente et tendances régionales. Bull. Fr. Pêche Piscic., 337/338/339, 63-74.

CHANGEUX T., PONT D., 1995. Current status of the riverine fishes of the French Mediterranean basin. Biological Conservation, 72, 137-158. 
CHESSEL D., THIOULOUSE J., HANAFI M., 1997. STATIS et valeurs typologiques des groupes faunistiques. Documentation thématique ADE-4 "Analyses multivariées et expression graphique des données environnementales ", Vol. 5, section 1 , Université Lyon I, 30 p. (ftp://pbil.univ-lyon1.fr/pub/mac/ADE/ADE4/DocThemPDF/ Thema51.pdf)

CRESPIN L., BERREBI P., 1994. L'hybridation naturelle entre le barbeau commun et le barbeau méridional en France : compte rendu de dix années de recherche. Bull. Fr. Pêche Piscic., 334, 177-189.

DE LURY D.B., 1947. On the estimation of biological populations. Biometrics, 3, 145-167.

DOLÉDEC S., CHESSEL D., 1991. Recent developments in linear ordination methods for environmental sciences. Advances in Ecology, India, 1, 133-155.

FISCHER P., ECKMANN R., 1997. Spatial distribution of littoral fish species in a large European lake, Lake Constance, Germany. Archiv für Hydrobiologie, 140, 1, 91116.

FOUCART T., 1978. Sur les suites de tableaux de contingence indexés par le temps. Statistique et Analyse des données, 2, 67-84.

FOUCART T., 1983. Une nouvelle approche de la méthode STATIS. Revue de Statistique Appliquée, 31, 61-75.

GRANDMOTTET J.P., 1983. Principales exigences de 30 téléostéens dulcicoles vis-à-vis de l'habitat aquatique. Annales Scientifiques de l'Université de Franche-Comté Besançon, $4^{\circ}$ série, 4, 3-32.

HUET M., 1954. Biologie, profil en long et en travers des eaux courantes. Bull. Fr. Piscic., 175, 41-53.

HUGUENY B., 1990. Richesse des peuplements de poissons dans le Niandan (Haut-Niger, Afrique) en fonction de la taille de la rivière et de la diversité du milieu. Revue Hydrobiologique Tropicale, 23, 351-364.

ILLIES J., BOTOSANEANU M., 1963. Problèmes et méthodes de la classification et de la zonation écologique des eaux courantes, considérées surtout du point de vue faunistique. Verhandlunger der Internationalen Vereinigung für theoretische und angewandte Limnologie, 12, 1-52.

LAROCHE J., BARAN E., RASOANANDRASANA N.B., 1997. Temporal patterns in a fish assemblage of a semiarid mangrove zone in Madagascar. Journal of Fish Biology, 51, 3-20.

LEGER L., 1937. Economie biologique générale des cours d'eau alpins. Bulletin Fr. Piscic., 109, 5-13.

LOUIS M., BOUCHON C., BOUCHON-NAVARO Y., 1995. Spatial and temporal variations of mangrove fish assemblages in Martinique (French West Indies). Hydrobiologia, $295,275-284$.

NOY-MEIR 1., 1973. Data transformations in ecological ordination. I - Some advantages of non-centering. Journal of Ecology, 61, 329-341.

OBERDOFF T., GUILBERT E., LUCCHETTA J.C., 1993. Patterns of fish species richness in the Seine River basin, France. Hydrobiologia, 259, 157-167.

PERSAT H., BERREBI P., 1990. Relative ages of present population of Barbus barbus and Barbus meridionalis (Cyprinidae in southern France : preliminary considerations. Aquatic Living Resources, 3, 253-263.

THIOULOUSE J., CHESSEL D., 1987. Les analyses multi-tableaux en écologie factorielle. I - De la typologie d'état à la typologie de fonctionnement par l'analyse triadique. Acta CEcologica, CEcologia Generalis, 8, 4, 463-480.

THIOULOUSE J., CHESSEL D., DOLÉDEC S., OLIVIER J.M., 1997. ADE-4 : a multivariate analysis and graphical display software. Statistics and Computing, 7, 1, 75-83. 
VERNEAUX J., 1973. Cours d'eau de Franche-Comté Massif du Jura. Recherches écologiques sur le réseau hydrographique du Doubs. Essai de biotypologie. Thèse d'Etat, Besançon, $257 \mathrm{p}$.

VERNEAUX J., 1981. Les poissons et la qualité des cours d'eau. Annales Scientifiques de l'Université de Franche-Comté Besançon, $4^{\circ}$ série, 2, 33-41. 